\title{
Lensless EUV mask inspection for anamorphic patterns
}

\author{
Iacopo Mochi, Hyun-Su Kim, Atoosa Dejkameh, Ricarda Nebling, Dimitrios Kazazis, Uldis \\ Locans, Tao Shen, and Yasin Ekinci \\ Paul Scherrer Institut, Villigen, Switzerland
}

\begin{abstract}
Anamorphic mask design is necessary to overcome the geometrical limitation imposed by the reflective geometry used in EUV lithography projection systems and will be used in high-NA EUVL scanners. In this study, we demonstrate the capability of anamorphic imaging using CDI. We created anamorphic EUV masks with programmed defects and inspected it with RESCAN, a lensless microscope dedicated to EUV mask inspection.
\end{abstract}

Keywords: CDI, EUV, anamorphic, lensless, metrology, mask inspection

\section{INTRODUCTION}

RESCAN (reflective-mode EUV mask scanning lensless imaging microscope) is a synchrotron-based actinic pattern inspection platform operating at the Swiss Light Source. It was developed to demonstrate the potential of coherent diffraction imaging (CDI) for actinic patterned mask inspection (APMI) on EUV reticles for current and future lithography nodes. RESCAN was used to demonstrate the inspection of amplitude and phase defects on random, logic-like, pattern with a sensitivity of $50 \times 50 \mathrm{~nm}$ and beyond. Through-pellicle inspection in vacuum and in low-pressure $\mathrm{H}_{2}$ atmosphere has also been successfully performed. ${ }^{1,2}$

The future of high-NA EUV lithography will rely on anamorphic mask designs to overcome the fundamental geometrical limitations imposed by the reflection configuration used in EUV scanners. ${ }^{3}$ Anamorphic lithography and metrology platforms based on conventional optics may require a new design of their projection or imaging systems. Because RESCAN is a lensless microscope, the transition to anamorphic pattern inspection requires only a simple modification of the image reconstruction procedure.

To verify the ability of RESCAN to effectively detect defects on anamorphic mask layouts, we created a reticle sample with programmed phase and amplitude defects in anamorphic random patterns. Amplitude defects range in size from $200 \mathrm{~nm}$ to $20 \mathrm{~nm}$ (on mask). The phase defects are realized with titanium pillars buried under the multilayer. The smallest bump has a height of $1.7 \mathrm{~nm}$ and a lateral size of $50 \mathrm{~nm}$ We inspected the sample with RESCAN mapping out the programmed defects and investigated the impact of the anamorphic geometry on the requirements for efficient and reliable image reconstruction.

\section{MATERIALS AND METHODS}

\subsection{Programmed defect anamorphic sample}

In order to test the ability of RESCAN to detect defects on anamorphic patterns, we created a dedicated sample with programmed amplitude and phase defects. The sample layout, shown in figure 1, includes a collection of randomly positioned structures with $1 / 2$ aspect ratio. The critical dimension (CD) is $400 \mathrm{~nm}$ along the horizontal direction and $200 \mathrm{~nm}$ along the vertical direction. The sample substrate consists of a silicon wafer with a $100 \mathrm{~mm}$ diameter, thickness of $0.625 \mathrm{~mm}$ and a surface roughness $\sigma<0.15 \mathrm{~nm} \mathrm{rms}$. The programmed phase defects were obtained creating titanium pillars with different heights on the wafer surface before the multilayer deposition. The local deformation of the multilayer induces a local phase variation in the reflected EUV beam. The structure of the multilayer is described in table 1 and it is designed to have a high reflectance $(R>65 \%)$ from $0^{\circ}$ to $12^{\circ}$ at $13.5 \mathrm{~nm}$ wavelength.

Further author information: E-mail: iacopo.mochi@psi.ch

Extreme Ultraviolet (EUV) Lithography XII, edited by Nelson M. Felix,

Anna Lio, Proc. of SPIE Vol. 11609, 116090M - @ 2021 SPIE

CCC code: $0277-786 \mathrm{X} / 21 / \$ 21 \cdot$ doi: $10.1117 / 12.2584518$

Proc. of SPIE Vol. 11609 116090M-1 


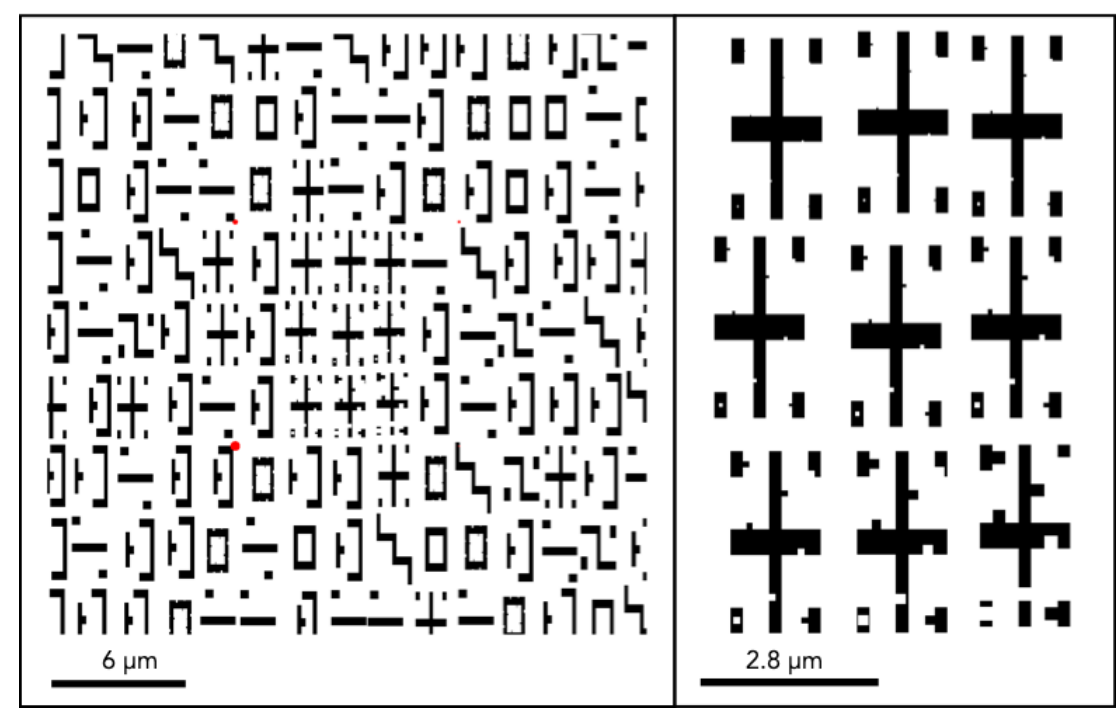

Figure 1. The sample includes four regions with anamorphic structures with aspect ratios of 2:1 and 1:2, with programmed defects and without. A. Vertically stretched layout. The central region includes an array of programmed defects. The red dots represent buried Ti pillars with a diameter ranging from $50 \mathrm{~nm}$ to $400 \mathrm{~nm}$. B. Detailed layout of the programmed defect region with intrusion and extrusion defects with size ranging from $20 \mathrm{~nm}$ to $200 \mathrm{~nm}$.

\begin{tabular}{c|c|c|c} 
Material & Thickness $[\mathrm{nm}]$ & $\mathrm{n}$ & $\mathrm{k}$ \\
\hline \hline $\mathrm{Si}$ & 2.506 & 0.99932 & -0.00183 \\
$\mathrm{MoSi} 2$ & 0.802 & 0.9693 & -0.00433 \\
$\mathrm{Mo}$ & 1.904 & 0.92108 & -0.00644 \\
MoSi2 & 1.844 & 0.9693 & -0.00433
\end{tabular}

Table 1. The multilayer coating of the sample consists of 40 Mo-Si bi-layers. MoSi2 intermixing regions are present between the molybdenum and silicon layers. The refraction index $n$ and the extinction coefficient $k$ for a wavelength $\lambda$ of $13.5 \mathrm{~nm}$ are shown for every layer.

A hydrogen silsesquioxane (HSQ) layer with a thickness of $140 \mathrm{~nm}$ was deposited on top of the multilayer and patterned with e-beam lithography to generate a logic-like layout with an array of programmed absorber defects. A detail of the layout is shown in figure 1. The layout includes anamorphic patterns with aspect ratios of 2:1 and 1:2. The choice of HSQ as absorber gives us the flexibility to create custom reticle samples in house, but, to achieve the same contrast, it requires an absorber layer twice as thick as in industrial EUV masks where TaNB is used. This increases the impact of shadowing and other mask 3D effects.

\subsection{RESCAN configuration}

RESCAN is a lensless microscope specifically designed to investigate the potential of coherent diffraction imaging techniques for EUV mask metrology. Its optical layout is shown in figure 2. The sample is illuminated by a focused EUV beam at an angle of incidence of $6^{\circ}$ from its normal. The illumination NA for this experiment was 0.002. The illumination beam has a wavelength $\lambda=13.5 \mathrm{~nm}$ and a bandwidth $\lambda / \Delta \lambda=1500$. The shape of the illumination spot on the sample can be approximated with an Airy pattern with a first minimum radius of $3.96 \mathrm{\mu m}$. The photon flux on the sample is $\sim 10^{12}$ photon/s. The diffracted light is collected by a CCD detector at a distance of $62 \mathrm{~nm}$ (Princeton Instruments MTE2-2048 pix). The resolution of the microscope is determined by the numerical aperture defined by the collection angle of the detector. In this case we used a portion of the CCD of $1900 \times 1900$ pix. The pixel size is $13.5 \mu \mathrm{m}$ and the corresponding NA is 0.207. Following the Rayleigh criterion, the resolution of the reconstructed image is therefore $1.22 \lambda / 2 \mathrm{NA}=40 \mathrm{~nm}$.

To take advantage of the anamorphic mask design, we installed the sample so that the light's incidence plane was perpendicular to the direction of the $2 \times$ structures (the horizontal axis in figure 1 ). 


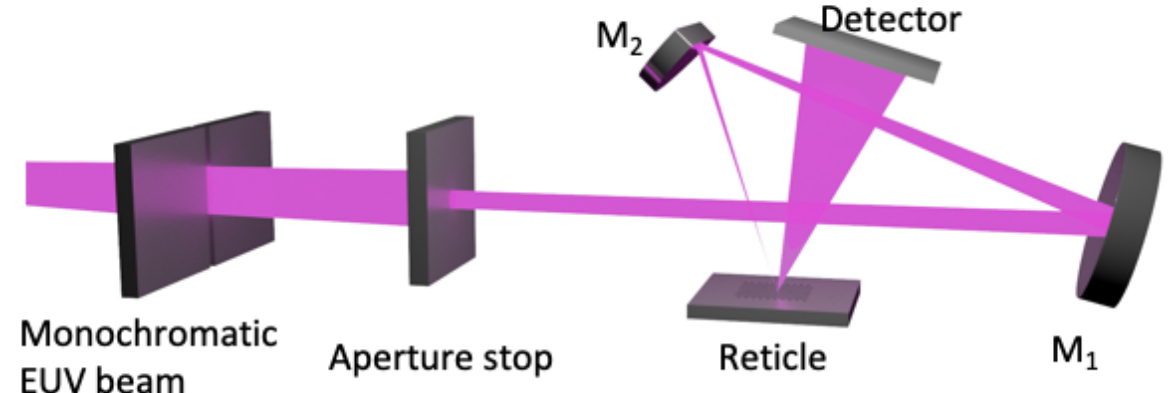

Figure 2. Optical layout of the RESCAN microscope. The EUV synchrotron beam, horizontally focused on the exit slit of a grating monochromator, is shaped by a circular aperture stop with a variable diameter. The beam is focused on the surface of the reticle sample by a toroidal mirror $\left(M_{1}\right)$ and by a flat folding mirror $\left(M_{2}\right)$. The diffracted light is collected by a detector at a distance of $62 \mathrm{~mm}$ from the sample.

\subsection{EUV reflection ptychography}

Ptychography, the image reconstruction method used in RESCAN, is one of the most robust scanning CDI techniques. ${ }^{4}$ It consists in collecting a series of diffraction pattern images while scanning the sample with a coherent illumination probe. For each scanning position, there must be some amount of probe overlap (from about $60 \%$ to $85 \%){ }^{5}$ The information redundancy thus produced allows to reconstruct the amplitude and the phase of the sample. Ptychography can be implemented in several ways ${ }^{6}$ and for the current experiment we used an hybrid algorithm combining the difference map ${ }^{7}$ and the rPIE approach. ${ }^{8}$ Both these methods rely on the fast Fourier transform algorithm to model the propagation of the electromagnetic field from the sample to the detector plane and back in an iterative manner. While for low-NA ptychography in transmission mode this is a fairly straightforward approach, in our case, it is important to consider the distortion effect on the measured diffraction patterns generated by RESCAN's off-axis reflection configuration. ${ }^{9}$ Even at a modest angle of incidence, with a detector NA of 0.2 the diffraction pattern detected on the sensor plane has a significant deviation from the spherical mapping modelled by the FFT-based propagation and causes a significant resolution loss in the reconstructed images. ${ }^{2}$ For this reason, we pre-processed all the collected diffraction pattern to remove the conical diffraction distortion.

\section{ANAMORPHIC MASK INSPECTION}

We scanned two areas of the sample containing the anamorphic pattern shown in figure 1 and an identical one with the opposite aspect ratio. The scan pattern covers an area of $36 \pi \mu^{2}$ and includes 106 sample positions with a step size of $1 \mu \mathrm{m}$ corresponding to a $68 \%$ overlap between the probes. The diffraction pattern we collected were scaled and corrected to remove the effect of the conical diffraction distortion induced by the 6 degree illumination configuration. ${ }^{2,9}$ The average corrected diffraction patterns for the two areas of the sample are shown in figure $3 \mathrm{~A}$ and $\mathrm{B}$. When the illumination incidence plane is perpendicular to the direction of the features with the largest CD (Fig. 3A), the diffraction intensity distribution lying on the incidence plane is compressed. This helps to overcome the geometrical limit set by the illumination optics and the reflectance loss caused by the multilayer for diffraction angles larger than 12 degrees. ${ }^{10}$ The opposite behavior is observed for a pattern with the inverse aspect ratio (Fig. 3B). Here the diffraction pattern extends further along the horizontal direction and gets partially shadowed by the multilayer reflectance limit as can be seen in the right side of the figure.

In a conventional anamorphic imaging optical system, the magnification is different along the vertical and horizontal direction which is usually achieved with separate optical elements for the two axes. In a lensless 


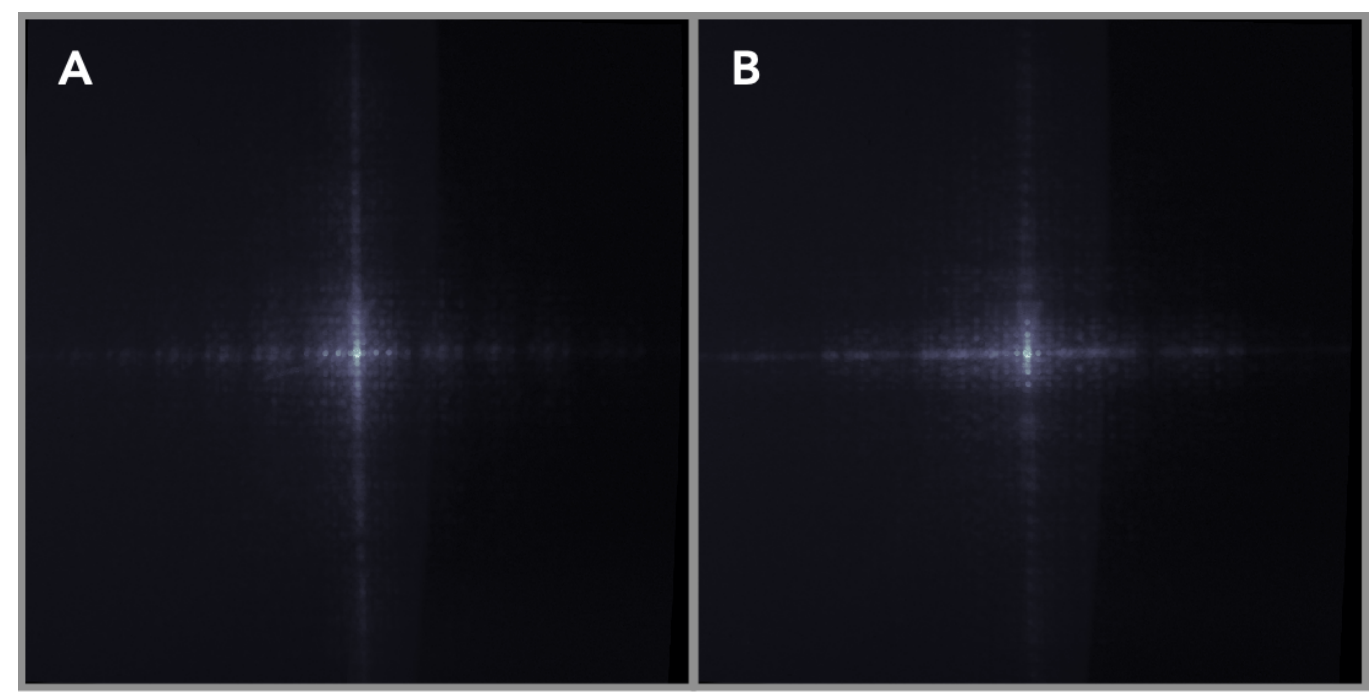

Figure 3. A. Average diffraction spectrum from the scan performed over the anamorphic sample with the illumination beam perpendicular to the direction of the features with the larger CD. B. Average diffraction spectrum from the scan performed over the anamorphic sample with the illumination beam perpendicular to the direction of the features with the smaller CD.

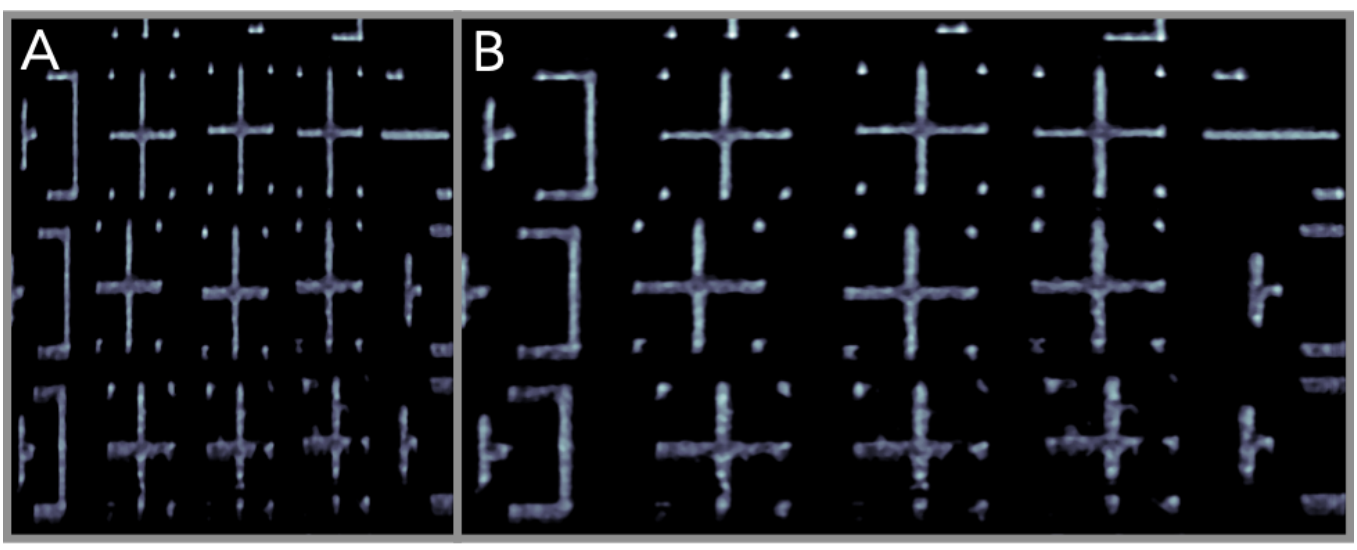

\section{$2.8 \mu \mathrm{m}$}

Figure 4. A. Detail of the reconstruction of the anamorphic sample. B. Detail of the reconstruction of the anamorphic sample after padding the diffraction data to correct the aspect ratio.

system, this can be easily done by remapping the diffraction data on an new grid. In practice, if we want to obtain an isomorphic image from a mask with an aspect ratio of $2: 1$, we can apply a Hann window ${ }^{11}$ and pad the original diffraction patterns with zeros along the axis that needs to be stretched. The result of this operation is shown in Figure 4. Here, we reconstructed an image of the sample corresponding to the diffraction pattern shown in figure $3 \mathrm{~A}$ without padding the data $(\mathrm{A})$ and with a zero padding to double the size of the detector in the horizontal direction (B).

When we review the defects, it might be useful to image the mask with the aspect ratio of the image projected on the wafer. In this case, however, the defects will be stretched along the axis with the higher magnification. In principle, the mask inspection process does not require an isomorphic image since the main goal is to determine the location of mask defects. The defects show in figure 5 were detected on the anamorphic image without any modification of the aspect ratio. Here we observed that RESCAN can detect defects as small as $50 \times 50 \mathrm{~nm}$ with a $50 \%$ contrast. 

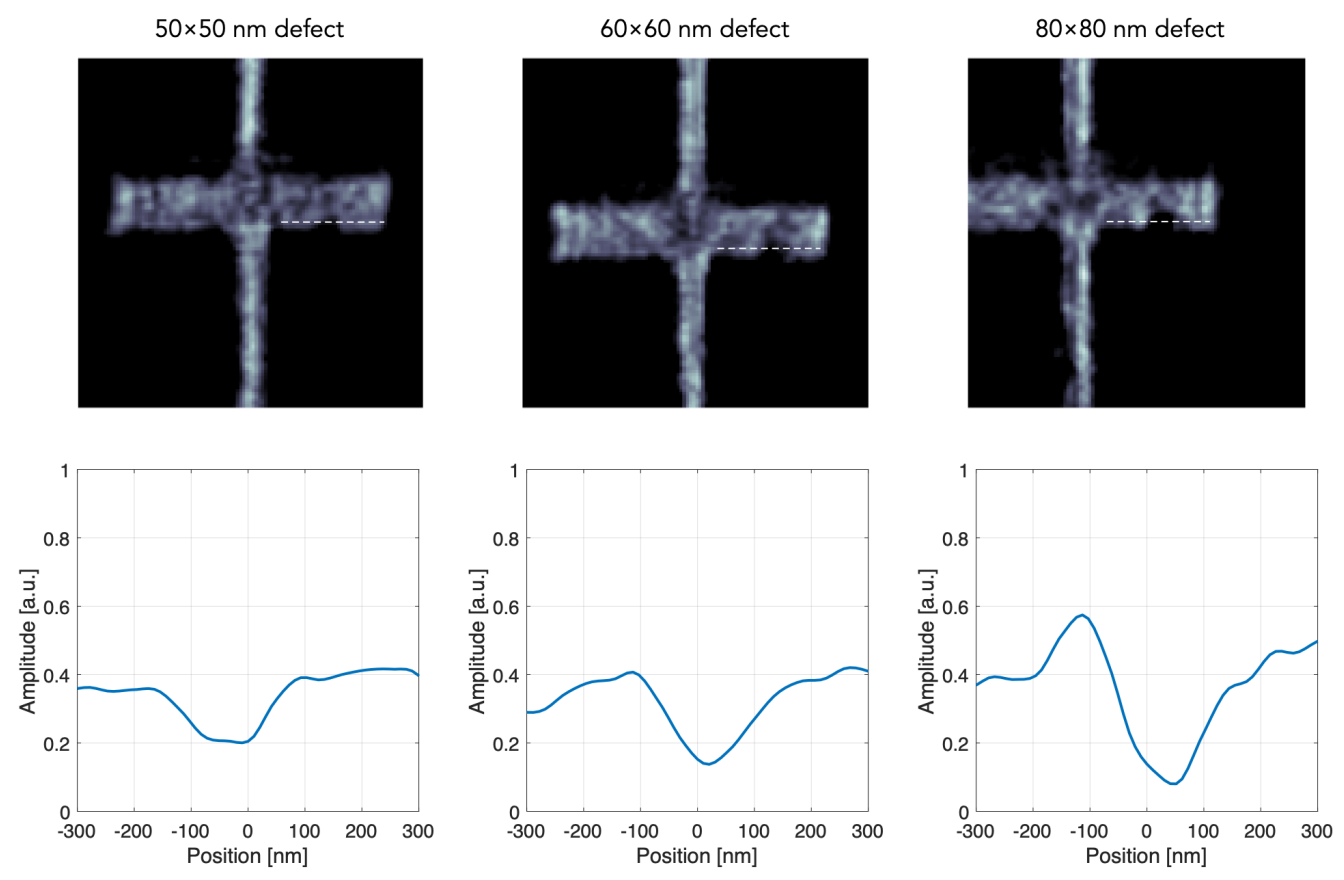

Figure 5. Detail of the reconstruction showing the location of three intrusion defects. The measured amplitude along the dashed lines showing the defect signature is shown below the respective images.

\section{CONCLUSIONS}

We created a dedicated EUV mask test sample to investigate the capability of the RESCAN microscope to perform APMI on anamorphic layouts. The sample absorber is HSQ with a thickness of $140 \mathrm{~nm}$. The advantage of a lensless approach to anamorphic mask inspection and review is that it requires only a simple and computationally inexpensive modification on the image reconstruction algorithm. We presented the first reconstruction results showing that RESCAN can detect amplitude defects as small as $50 \times 50 \mathrm{~nm}$ (on mask) on anamorphic samples. RESCAN is currently undergoing an optics upgrade to increase its resolution and to enable structured pupil illumination, but to fully explore its potential for mask inspection, we are looking into the possibility to manufacture or procure a sample with a thinner absorber like TaBN for which shadowing and mask 3D effects will be less prominent.

\section{ACKNOWLEDGMENTS}

The authors wish to thank the team of the XIL beamline of the Swiss Light Source, Michaela Vockenhuber, Markus Kropf and José Gabadinho for their technical support. This project has received funding from the Electronic Component Systems for European Leadership Joint Undertaking under grant agreement No 783247TAPES3. This Joint Undertaking receives support from the European Union's Horizon 2020 research and innovation program and Netherlands, Belgium, France, Germany, Israel.

\section{REFERENCES}

[1] Mochi, I., Timmermans, M. Y., Gallagher, E. E., Mariano, M., Pollentier, I., Rajendran, R., Helfenstein, P., Fernandez, S., Kazazis, D., and Ekinci, Y., "Experimental evaluation of the impact of carbon nanotube euv pellicles on reticle imaging," Journal of Micro/Nanolithography, MEMS, and MOEMS 18(1), $1-7-7$ (2019). doi:10.1117/1.JMM.18.1.014002.

[2] Kim, H., Locans, U., Nebling, R., Dejkameh, A., Kazazis, D., Ekinci, Y., and Mochi, I., "High resolution and uniform image reconstruction in a large field-of-view for EUV actinic mask review," in [Photomask 
Technology 2020], Preil, M. E., ed., 11518, 146 - 152, International Society for Optics and Photonics, SPIE (2020).

[3] Kneer, B., Migura, S., Kaiser, W., Neumann, J. T., and van Schoot, J., "EUV lithography optics for sub9nm resolution," in [Extreme Ultraviolet (EUV) Lithography VI], II, O. R. W. and Panning, E. M., eds., 9422, 461 - 470, International Society for Optics and Photonics, SPIE (2015).

[4] Helfenstein, P., Rajeev, R., Mochi, I., Kleibert, A., Vaz, C. A. F., and Ekinci, Y., "Beam drift and partial probe coherence effects in EUV reflective-mode coherent diffractive imaging," Opt. Express 26, 12242-12256 (Apr 2018). [doi:10.1364/OE.26.012242].

[5] Bunk, O., Dierolf, M., Kynde, S., Johnson, I., Marti, O., and Pfeiffer, F., "Influence of the overlap parameter on the convergence of the ptychographical iterative engine," Ultramicroscopy 108(5), 481-487 (2008).

[6] Li, P. and Maiden, A. M., "Ten implementations of ptychography," Journal of Microscopy 269(3), 187-194 (2018).

[7] Thibault, P., Dierolf, M., Bunk, O., Menzel, A., and Pfeiffer, F., "Probe retrieval in ptychographic coherent diffractive imaging," Ultramicroscopy 109(4), 338 - 343 (2009).

[8] Maiden, A., Johnson, D., and Li, P., "Further improvements to the ptychographical iterative engine," Optica 4, 736-745 (Jul 2017). doi:10.1364/OPTICA.4.000736.

[9] Gardner, D. F., Zhang, B., Seaberg, M. D., Martin, L. S., Adams, D. E., Salmassi, F., Gullikson, E., Kapteyn, H., and Murnane, M., "High numerical aperture reflection mode coherent diffraction microscopy using off-axis apertured illumination," Opt. Express 20, 19050-19059 (Aug 2012).

[10] Mochi, I., Kim, H.-S., Locans, U., Dejkameh, A., Nebling, R., Kazazis, D., and Ekinici, Y., "Illumination control in lensless imaging for EUV mask inspection and review," in [Extreme Ultraviolet (EUV) Lithography $X I]$, Felix, N. M. and Lio, A., eds., 11323, 372 - 380, International Society for Optics and Photonics, SPIE (2020).

[11] Harris, F. J., "On the use of windows for harmonic analysis with the discrete fourier transform," Proceedings of the IEEE 66(1), 51-83 (1978). 